\title{
La feminización de la pobreza: conceptualizaciones actuales y potencialidades analíticas
}

\author{
Paula Lucía Aguilar \\ Universidad de Buenos Aires (UBA)
}

La feminización de la pobreza: conceptualizaciones actuales y potencialidades analíticas

Resumen: Este artículo se propone describir, a partir de una revisión bibliográfica, algunos ejes de problematización posibles sobre la noción de "feminización de la pobreza" y su utilización en el campo de las políticas sociales. Sitúa, brevemente, los antecedentes del concepto de feminización a partir de su emergencia, en el contexto de los EEUU, hacia fines de los años 1970 y su expansión como diagnóstico para América Latina durante los años 1990. Luego se describen algunas definiciones representativas de sus usos, sentidos circulantes y la relación con el concepto de jefatura femenina del hogar. Para concluir, se describen dos lecturas alternativas presentes en la bibliografía revisada, que permitirán acercarse a la problemática de la pobreza desde una perspectiva más compleja, y que, a la vez, considere al género de modo relacional e integrado a los procesos sociales más amplios.

Palabras clave: Género. Pobreza. Discurso. Política social.

\section{A feminização da pobreza: conceitualizações atuais e potencialidades analíticas}

Resumo: Este artigo propõe-se a descrever, a partir de uma revisão bibliográfica, alguns possíveis eixos da problemática sobre a noção de "feminização da pobreza" e sua utilização no campo das políticas sociais. Situa, brevemente, os antecedentes do conceito de feminização a partir de sua emergência, no contexto dos Estados Unidos, no final dos anos 1970 e sua expansão como diagnóstico para América Latina durante os anos 1990. Depois, descrevem-se algumas definições representativas de seus usos, sentidos circulantes e a relação com o conceito de chefatura feminina do lar. Para concluir, são apresentadas duas leituras alternativas presentes na bibliografia revisada, que irão permitir uma aproximação à problemática da pobreza desde uma perspectiva mais complexa e que, por sua vez, considere este gênero de modo relacional e integrado aos processos sociais mais amplos.

Palavras-chave: Gênero. Pobreza. Discurso. Política social.

\section{The Feminization of Poverty: Current Theories and Analytic Potential}

Abstract: This article conducts a bibliographic review to then describe some possible lines of questioning of the concept "feminization of poverty" and its use in the field of social policy. It briefly situates the antecedents of the concept of feminization based on its emergence in the U.S. in the late 1970s and its expansion to Latin America in the 1990s. It then describes some definitions that are representative of its current uses and meanings and the relationship with the concept of female headed household. It concludes by describing alternative readings found in the bibliography reviewed, that allow approaching the problem of poverty from a more complete perspective, and which in turn consider gender in a relational manner integrated to broader social processes.

Key words: Gender. Poverty. Discourse. Social policy. 


\section{Presentación}

En este artículo me propongo delinear, a partir de una revisión bibliográfica, algunos posibles ejes de problematización acerca de la noción de "feminización de la pobreza" y su utilización en el campo de las políticas sociales. La necesidad de examinar esta categoría surge del trabajo de investigación en curso ${ }^{1}$ y de la observación de la creciente presencia que su utilización como diagnóstico ha cobrado desde mediados de la pasada década de los 90, tanto en el discurso acerca de la pobreza como en la orientación de las estrategias de intervención.

La utilización de la categoría "feminización de la pobreza" dista de ser uniforme o de referirse siempre a los mismos procesos históricos sociales. En general, es utilizada sin mayores aclaraciones respecto de su significado específico, para aludir al "predominio creciente de las mujeres entre la población empobrecida" (MURGUIALDAY, 2006) constituyendo, a la vez, una expresión "concisa y polivalente" (MOLYNEUX, 2006). Simultáneamente, debido a su contundencia y generalizada aceptación como diagnóstico de problemas sociales, la afirmación de su existencia y la necesidad de adoptar medidas que la tengan en cuenta como un objetivo primordial, constituye una de las muchas formas en que la perspectiva de "género" se ha ido incorporando al campo más amplio del debate sobre el desarrollo y las políticas sociales. Eficaz para visibilizar y potenciar la agenda pública sobre los múltiples problemas que hacen a la desigualdad de las condiciones de vida, los derechos y las oportunidades entre varones y mujeres; la generalidad de esta categoría opaca algunos de los procesos que busca designar con especificidad. Esta falta de claridad se extiende también al debate sobre su efectiva captación estadística (MEDEIROS; COSTA, 2008; DAMIAN, 2003).

En este marco, considero pertinente problematizar la noción de "feminización de la pobreza", a partir de la descripción de algunos de sus usos más frecuentes, y dar cuenta de las dificultades y potencialidades analíticas que conlleva para el estudio de políticas sociales que tengan en cuenta las desigualdades de género, en toda su amplitud. Más aun, si se considera que las categorías sobre las cuales se construyen los diagnósticos relacionados con los problemas sociales son parte constitutiva de las posibles respuestas, y que cada una de las respuestas ensayadas van definiendo las formas complejas, (simbólicas y materiales), que adoptan las condiciones de reproducción social (GRASSI, 2003).

El presente artículo se organiza en cuatro apartados. En el primero, se explican, brevemente, los antecedentes del concepto de feminización de la pobreza, desde su emergencia en el contexto de los EEUU hacia fines de los años 1970, hasta su expansión como diag- nóstico para América Latina durante los años 1990. En segundo lugar, se describen algunas definiciones representativas de sus utilizaciones analíticas y sentidos circulantes. Después, se realiza un repaso de las condiciones de aceptación y generalización de este diagnóstico como un impulso para la introducción de temáticas de género, en el discurso de las políticas sociales, sobre todo, a través de su relación con el concepto de jefatura femenina del hogar y se exponen algunos de los supuestos que subyacen a la utilización de este último. Para concluir, se describen las lecturas alternativas, presentes en la bibliografía revisada, que permitirán acercarse a la problemática de la pobreza desde una perspectiva compleja, y que a la vez, considere al género de modo relacional e integrado a los procesos sociales más amplios.

\section{Antecedentes}

El concepto que nos ocupa, "feminización de la pobreza", fue acuñado en los EEUU hacia fines de la década del 70. La bibliografía consultada (CHANT, 2003a; MOGHADAM, 2005) coincide en atribuir la primera mención del mismo a un trabajo de la investigadora Diana Pearce, de 1978, titulado: The feminization of poverty: Women, work, and welfare. La atención de ese trabajo se centraba particularmente en la descripción, en términos estadísticos, que se refería al aumento de los hogares encabezados por mujeres en los EEUU (que pasaron del $10.1 \%$ en 1950 para un $14 \%$ en 1976 , resultando en un $40 \%$ de aumento) y la correlación de ese hecho con el deterioro de sus condiciones de vida, en términos de pobre$\mathrm{za}$ (por ingresos). Ese aumento se desarrollaba en un contexto de transformaciones demográficas tales como el aumento de la expectativa de vida de las mujeres y el aumento de los divorcios, entre otras. De acuerdo con Diana Pearce: "Mientras que muchas mujeres son pobres porque viven en hogares pobres encabezados por varones, un creciente número de mujeres (se) están empobreciendo por propio derecho". Partiendo de esta base, se pregunta: “¿Cuáles son las consecuencias de ser mujer que resultan en tasas más altas de pobreza?" (PEARCE, 1978). Para ello, analiza la posición desigual en que las mujeres se encuentran ante la posibilidad de obtención de ingresos (tanto a través de su participación en el mercado de trabajo como en la seguridad social y los aportes familiares), y las formas en que los programas de ayuda gubernamental ${ }^{2}$ reproducían e institucionalizaban la desventajas de las mujeres frente a las situaciones de pobreza, considerando estos programas como "Workhouses sin paredes" (PEARCE, 1978) que profundizaban las desigualdades.

En los EEUU, la noción de "feminización de la pobreza" tuvo su desarrollo más importante en la 
década del 80, generando un cierto "clima de investigación" académica sobre las problemáticas que la categoría tornó visibles, en el contexto del debate impulsado por las reformas neoconservadoras de la política social de principios de los 80 , relacionadas con la histórica discusión acerca de la dependencia estatal concentrada en la figura de las mujeres sin pareja y a cargo de sus hijos (O'CONNOR, 2001; FRASER, 1997). Estas primeras formulaciones fueron objeto de críticas, ya que el acento puesto sólo en la feminización no tomaba en cuenta los aspectos de nacionalidad, raza y clase como explicativos y complementarios en la producción de la desigualdad (MOHANTY, 1987).

La denominada "década de la mujer", promovida por la ONU y sus organismos conexos (1975-1985), tuvo como efecto el impulso del análisis y la investigación de la situación de la mujer, en general, y de la "jefa de familia/hogar", en particular. Se realizaron, en ese período, numerosas investigaciones sobre la "mujer rural" (LEON; DEERE, 1986) y sobre la participación de la mujer en el desarrollo (entendido como crecimiento y modernización económica). En el prólogo de sus trabajos de 1986, donde realizan un balance de la "década de la mujer", las investigadoras Leon y Deere (1986, p. 11) advierten, tempranamente, sobre las consecuencias de la llamada crisis de la deuda, cuyos efectos habían "facilitado que se empiecen a reconocer los múltiples papeles que cumple la mujer, porque, a menudo, es ella la que sirve de colchón amortiguador de las presiones cada vez más fuerte de la crisis". Sin embargo, sostienen que "el punto de discusión no reside tan solo en que las mujeres participen de la solución de la crisis, sino también en que condiciones se define su participación y quien las define". Argumentos de notable actualidad, que resumen debates aún abiertos en cada diseño de la política social.

A partir de los años 90, la "feminización de la pobreza" se extiende con fuerza en el léxico del desarrollo y las políticas sociales a nivel global, circulando en el discurso y como requisito de financiamiento de diversos organismos internacionales ${ }^{3}$. Es en la IV Confe- rencia de las Naciones Unidas sobre la Mujer, realizada en Beijing en 1995, donde se afirma que el 70\% de los pobres del mundo eran mujeres y que la erradicación de la carga "persistente y 'cada vez mayor' de la pobreza que enfrentan las mujeres" fue incorporada como una de las 12 áreas críticas de la Plataforma de Acción acordada en la conferencia. Esta idea posiciona con énfasis a la mujer (más que las relaciones de género y en muchos casos reemplazando una categoría por otra) en el discurso de los foros internacionales respecto a la pobreza. Sin embargo, de acuerdo con el balance que realiza la antropóloga Jeanine Anderson, los resultados de la incorporación del concepto de feminización fueron limitados en sus potencialidades. Esa incorporación no fue seguida, necesariamente, "por un esfuerzo sostenido en la indagación empírica o en la elaboración teórica alrededor de las situaciones que de pronto fueron visibilizadas" (ANDERSON, 2003, p. 3). De acuerdo con su análisis:

En la práctica, su impacto fue bastante más limitado. Colocó en la mira dos problemáticas: 'las mujeres jefas del hogar' y la 'maternidad precoz' (frecuentemente vinculada a la condición de madre sola, jefa de un hogar incipiente o de una unidad subsidiaria dentro de otro hogar). Indujo a la desagregación estadística por sexo y creó un clima para el ingreso posterior de una preocupación por 'las voces' de las víctimas, la percepción de los actores y el uso de métodos cualitativos para describirlas (ANDERSON, 2003, p. 2).

Chant, por su parte, plantea que la generalización y el desplazamiento, desde la definición de principios de la década del 80, de la "feminización" como una tendencia en evolución a ser observada en los datos demográficos y económicos más cercanos a la conceptualización inicial de Diana Pearce, hacia la afirmación de la "feminización" como un hecho consumado y urgente sobre el que debe actuarse a través de intervenciones específicas, constituye un hecho fortuito (CHANT, 2005). Lejos de pensar en esta cristalización conceptual como claramente "fortuita", considero que es el efecto en conjunto de los procesos múltiples en los que la instalación de la "feminización" de la po- 
breza y sus definiciones operativas como un hecho consumado no se da en el vacío, sino que se configura sobre sentidos previos con respecto a los lugares socialmente construidos para mujeres y varones, sus condiciones de vida y la definición de estrategias de intervención sobre los problemas sociales hegemónicos en cada momento.

\section{Ambigüedad}

Mencionamos, en el inicio de este trabajo, que el concepto de feminización abarca contenidos múltiples. Un trabajo realizado por el PNUD resume los más comúnmente utilizados, como sigue:

a) un predominio de mujeres entre los pobres; b) el impacto no fortuito, con sesgo de género, de las causas de la pobreza; c) el reconocimiento de una tendencia direccional en la cual la representación desproporcionada de las mujeres entre los pobres está aumentando progresivamente (en este sentido, la feminización de la pobreza es un proceso, no simplemente un estado de cosas en una coyuntura particular); d) el grado de visibilidad de la pobreza femenina. En la medida que la unidad de análisis de los estudios e investigaciones son los hogares, no se consideran las reglas que rigen la distribución interna en una unidad doméstica, y que varían ampliamente según sociedades y culturas (PNUD, 1997, online).

Podemos notar en estas definiciones una convivencia de aspectos cuantitativos, relativos a la población y a la distribución de personas pobres clasificadas según su sexo (a), otras que describen tendencias y causalidades (b y c) y definiciones relacionadas con la visibilidad del fenómeno a estudiar. En su búsqueda por articular la medición estadística y clarificar conceptualmente la categoría, para lograr estudios comparativos, Medeiros y Costa proponen una aproximación analítica a su definición. Para estos autores, la "feminización" de la pobreza es "un cambio en los niveles de pobreza que muestra una tendencia en contra de las mujeres o de los hogares a cargo de mujeres" (MEDEIROS; COSTA, 2008, p. 2). La definición exacta, plantean, depende de dos preguntas secundarias y sencillas en su formulación, aunque muy difíciles de responder: ¿Qué es la pobreza? ¿Qué es la feminización? Aclaran que la palabra feminización connota una acción, "el proceso de volverse más femenino" o "más común o extendido entre las mujeres". Es este sentido de "proceso en curso" el que debe ser especificado en cada caso. Así, distinguen este "proceso" de lo que identifican como un estado: "la feminización de la pobreza no debería confundirse con el predominio de niveles más elevados de pobreza entre mujeres y hogares a cargo de mujeres" (MEDEIROS; COSTA, 2008). Por otra parte, plantean que pensarla como un estado habla de una descripción "estática" que supone cambios en el perfil de la pobreza, en lugar de cambios diferenciales en los niveles de pobreza entre los géneros.

Además de identificar la feminización en su dimensión de tendencia o proceso, se debe revisar el tipo de pobreza al que se hace referencia en estas afirmaciones (generalmente referidas a la pobreza por ingresos, por medio del análisis de agregados estadísticos). Sylvia Chant (2005) critica que sea el "ingreso" el principio fundamental en la consideración de la feminización, y que a pesar del enorme bagaje de producción académica sobre el carácter complejo, tanto de la pobreza definida en términos objetivos como subjetivos y en los cuales la asimetría de género ocupa un lugar importante, éste continúe siendo el criterio que predomine en la consideración y el uso de la categoría. Este énfasis en el ingreso provoca el descuido analítico de otras dimensiones de la pobreza y los aspectos relacionados con la discriminación de género (falta de acceso a recursos, crédito, tierras, ingresos) discriminación legal, política, cultural y religiosa. Estos aspectos permanecen invisibles, a menos que se expliquen las desigualdades específicas de modo cultural e históricamente situadas ${ }^{4}$.

Un último elemento que refuerza las imprecisiones es el uso generalizado de la categoría de género, como sinónimo y reemplazo "políticamente correcto" de la categoría mujer (de connotaciones biológicas y esencialistas); pero sin considerar el carácter relacional que analíticamente conlleva un análisis con perspectiva de género. En este sentido, el análisis de la "feminización" de la pobreza suele incluir el aumento de la proporción de mujeres entre la población pobre y, más específicamente, el numero de las jefas del hogar, sin considerar de que modo las relaciones jerárquicas entre varones y mujeres en el interior de los hogares, (no necesariamente encabezados por estas últimas), construyen estas condiciones desiguales de vida. La pregunta que subyace a esta utilización y que deberíamos intentar contestar es: ¿Qué implica, a respecto de los hombres y las relaciones de género, afirmar la feminización de la pobre$\mathrm{za}$ ?

\section{Focalización y jefatura del hogar: claves de la expansión del concepto}

Es importante destacar que la generalización del diagnóstico acerca de la creciente feminización de la pobreza ha tenido consecuencias importantes para la agenda del debate y los análisis de las políticas, a pesar de la amplitud de su definición y de la 
coincidencia entre los especialistas en señalar la ausencia de datos estadísticos que den cuenta cabal de la especificidad del proceso. La bibliografía consultada destaca un efecto político fundamental para el uso de este concepto: el diagnóstico de la feminización ha llamado la atención de los encargados del diseño de programas y políticas de un modo que há permitido incorporar y aumentar la posición de las demandas de las mujeres, en los ámbitos de discusión internacionales. En este sentido, aún sus más insistentes críticos, rescatan la fuerza como estrategia política para la incorporación de las temáticas “de género", en la agenda de diseño y financiamiento.

$\mathrm{Su}$ aceptación e incorporación a la agenda se debe, al menos parcialmente, al supuesto de la "reproducción intergeneracional de la pobreza". Así, la articulación entre problemáticas de género y pobreza que presenta la noción de feminización se vuelve "tentadora", en términos de eficiencia para el diseño de políticas, ya que sería la vía ideal para actuar simultáneamente sobre las condiciones de vida y sobre las demandas acerca de las desigualdades de género ${ }^{5}$. Además, en términos teóricos, ésta favorecería su expansión en los ámbitos gubernamentales y organismos internacionales porque articula estas acciones a partir de la idea de "inversión social", en la cual las mujeres destinatarias de la ayuda actuarían como inversión actual "eficiente" y con importantes rendimientos en el futuro (el bienestar de sus niños).

Esta generalización de la perspectiva de la "feminización" de la pobreza en el discurso está también asociada con la identificación de la pobreza como el principal problema social para ser atendido. Como se expuso anteriormente, ciertas definiciones de "feminización" la circunscriben y asocian al aumento de la pobreza en los hogares encabezados por mujeres, lo cual permite particularizar los criterios de focalización de programas, asimilándolos con los hogares "más pobres entre los pobres", según reza la fórmula del Banco Mundial. Así, la noción de "feminización" actúa dando por sentado que los hogares con jefatura femenina, en tanto suponen desigualdades persistentes, pueden actuar como criterio de focalización casi automático para identificar a los hogares

[...] más pobres entre los pobres, y al mismo tiempo, actuar sobre las desigualdades de género (asimilando género y mujer como categorías equivalentes). Sin embargo, es claro que el análisis de las jefaturas del hogar no pueden ni deben considerarse como un sustituto aceptable para el análisis de género y pobreza (LAMPIETTI; STALKER, 2000 apud CHANT, 2005, p. 2).

Es importante señalar que las políticas de disminución de la pobreza y de la desigualdad de género no son necesariamente una ecuación directa, y muchas veces, su asociación culmina en la instrumentalización (MOLYNEAUX, 2006, 2007). De este modo,

[...] algunos programas para combatir la pobreza reproducen patrones de discriminación, toda vez que las mujeres son usadas como prestadoras de servicios de asistencia social o familiar no pagadas o mal retribuidas (GODOY, 2004, p. 54).

El discurso sobre la feminización reificada como un hecho y vinculada directamente con la focalización en la jefatura femenina de los hogares refuerza los diagnósticos acerca de la pobreza (en tanto fenómeno) como un problema social aislado de sus causas estructurales y desvinculado del mercado de trabajo. Se evidencia una preocupación por las formas visibles del fenómeno a partir del diagnóstico de la feminización (expresado en el aumento de hogares con jefatura femenina), y no por el conjunto de causas a partir de las cuales los hogares que tienen esta característica, en particular, tienen por correlato necesario situaciones de desigualdad o vulnerabilidad para sus miembros. Así,

El énfasis en los hogares con jefatura femenina como los más pobres entre los pobres conlleva la impresión de que la pobreza se debe más a las características del hogar (que incluyen el estado civil o marital) de quien lo encabeza, que a los contextos macro sociales y económicos en los que se sitúan estos hogares (CHANT, 2003a, traducción propia).

A la poca disponibilidad de datos sobre pobreza desagregados por sexo y de los estudios longitudinales periódicos, que permitan realizar la evaluación de las situaciones a lo largo del tiempo, se suma la identificación de la jefatura femenina del hogar, no sólo como vulnerable en sí misma, sino como sinónimo asimilable a "todas" las mujeres y no prestando atención a otras variables en juego (como, por ejemplo, la edad o la presencia de niños pequeños), que efectivamente tienen gran influencia tanto a partir de la extensión de la expectativa de vida como de las brechas entre mujeres y varones jóvenes en la vida laboral. Esto redunda en una falta de atención a las diferencias entre las mujeres y la diversidad de condiciones de vida en las que puedan encontrarse.

Esta concentración en los hogares encabezados por mujeres, como un modo de analizar la feminización de la pobreza, puede mostrar como situaciones de victimización y de carencia, elecciones que en los términos de las condiciones de vida de esas mujeres pueden ser positivas. Los procesos que llevan a encabezar los hogares son muchos, y aunque es cierto que se verifica una precarización de las condiciones 
de vida, la pobreza no se deriva automáticamente de la condición de jefa del hogar, tal como lo sugieren algunas focalizaciones derivadas del diagnóstico de su "feminización". Las características de los hogares encabezados por mujeres y las situaciones que llevaron a ese resultado son heterogéneas y complejas 6 .

Medeiros y Costa destacan que medir los niveles de pobreza "entre las mujeres" y "entre los hogares a cargo de mujeres" no refleja la feminización de la pobreza. La jefatura del hogar tiene por objetivo demostrar lo que sucede con grupos específicos vulnerables de mujeres y sus familias. Los indicadores de pobreza "entre las mujeres" separan hombres y mujeres como individuos, e incluyen o excluyen de sus totales a los niños. Debemos reflexionar los modos en que la pobreza femenina y masculina están intrínsecamente relacionadas al nivel del hogar (MEDEIROS; COSTA, 2008). Es necesario recuperar el debate de los procesos intrahogar sin enfocar o deducir solo a partir de su jefatura, las características de sus condiciones de vida.

\section{Otras lecturas: feminización de la respon- sabilidad y estudio de sus causas}

Ahora bien, habiendo descripto el surgimiento y las articulaciones conceptuales que favorecieron la inclusión y aceptación de la noción de feminización de la pobreza como diagnostico, es importante considerar dos propuestas que buscan especificar su capacidad analítica, al tiempo que intenta retener la potencialidad política que permitió incluir las temáticas de género en la agenda pública.

Chant propone visibilizar para el análisis lo que llama la "feminización de la responsabilidad y la obligación", desde un contexto más amplio que tome en cuenta las condiciones materiales de vida y los múltiples procesos discriminatorios que deben ser abordados cuando se consideran las brechas de ingresos, las condiciones de trabajo y de vida entre varones y mujeres, que ubican a estas últimas en situaciones de pobreza. Sugiere la necesidad de considerar de que modo las mujeres se encuentran cada vez más en el "frente de batalla", y como la carga de la supervivencia familiar recae de manera desproporcionada sobre ellas. Rescatar la dimensión de la "feminización de la responsabilidad", de acuerdo con la autora, tiene como objetivo transmitir la idea "de que las mujeres se encuentran asumiendo una mayor responsabilidad en hacerle frente a la pobreza" (CHANT, 2005 p. 214) y que esta responsabilidad es invisibilizada y en muchos casos "instrumentalizada" por el diseño de las políticas.

Otra propuesta, para hacer más fructífero el concepto de "feminización", es el acento que
Medeiros y Costa proponen a partir de pensar una "feminización de las causas" de la pobreza. Su trabajo considera que es preferible especificar como influye en la vida social la feminización de las "causas" de la pobreza, o dicho de otro modo, de que formas actúan las "jerarquías" de género en la producción y reproducción de las situaciones de pobreza, diferenciando esta perspectiva del concepto de la feminización como "estado" o "resultado" y poniendo énfasis sobre su carácter de proceso social:

\section{La reacción común, en términos de políticas basadas en la feminización de la pobreza, consiste en centrarse en las mujeres, tiende a traducirse en intervenciones orientadas a un solo grupo y/o una sola problemática, y que tienen una capacidad limi- tada para desestabilizar al mercado laboral y otras instituciones (MEDEIROS; COSTA, 2008, p. 120).}

Tanto la propuesta de pensar en una feminización de las causas como la perspectiva de las responsabilidades, ubica a la feminización como un proceso entramado en relaciones sociales más amplias y supone una categoría de género que recupera su carácter relacional y, por tanto, en permanente construcción. Ahora bien, si aceptamos que la articulación de las categorías de género con las políticas para la disminución de la pobreza, en términos de discurso político, coadyuvan en garantizar ciertos recursos para las mujeres y promover el reconocimiento de algunas de sus demandas en la esfera política; el interrogante surge con fuerza "¿por qué abandonar un término que ha demostrado ser tan fructífero?" (CHANT, 2005, p. 209). Sin negar los avances realizados hasta el momento, en la instalación del género como perspectiva insoslayable para la comprensión de los problemas sociales, es necesario tener en cuenta que las categorías con las cuales los diagnósticos se conforman están en permanente disputa y que para que esa utilización estratégica de la "feminización" se concrete efectivamente, en políticas que superen la mera focalización en las jefas del hogar, es necesario preguntarse de que manera una idea como la feminización y sus múltiples sentidos pueden estar actuando en varias estrategias discursivas al mismo tiempo, orientando políticas en un sentido que dista de ampliar las protecciones, los derechos y las garantías ciudadanas. Es a través de esta interrogación permanente que ponemos a resguardo su carácter estratégicamente fructífero.

\section{Referencias}

ANDERSON, J. Diálogos con los conceptos de "conceptos claves". In: DESAFÍOS CONCEPTUALES DE LA POBREZA DESDE UNA PERSPECTIVA DE GÉNERO, 
Primer Módulo. 2003. Disponible en: 〈www.eclac.org/mujer/ reuniones/pobreza/PonenciaJeanineAnderson.doc $>$. Acceso en: 12 mar. 2010.

CHANT, S. Dangerous Equations? How Female-headed Households became the Poorest of the Poor: Causes, Consequences and Cautions. In: WORKSHOP FEMINIST FABLES AND GENDER MYTHS: Repositioning Gender in Development Policy and Practice, 2-3 Jul., 2003. Sussex: Institute of Development Studies, 2003.

¿Cómo podemos hacer que la "feminización de la pobreza" resulte más relevante en materia de políticas? ¿Hacia una "feminización de la responsabilidad y la obligación"? In: MORA, L.; MORENO RUIZ, M. J.; ROHRER, T. (Ed.). Cohesión social, políticas conciliadoras y presupuesto público: una mirada desde el género. México: Unfpa, 2005, p. 201-234.

DAMIAN, A. Tendencias recientes de la pobreza con enfoque de género en América Latina. Papeles de Población, Toluca: Universidad Autónoma del Estado de Toluca, n. 38, p. 26-27, oct./dic., 2003.

FRASER, N. Iustitia interrupta. Reflexiones críticas desde la posición "post-socialista". Santa Fé de Bogotá: Siglo del Hombre Editores, Universidad de los Andes, 1997.

GODOY, L. Entender la pobreza desde la perspectiva de género. Santiago de Chile: Cepal, 2004. (Serie Mujer y Desarrollo, n. 52).

GRASSI, E. Política y cultura en la sociedad neoliberal. La otra década infame I. Buenos Aires: Espacio Editorial, 2003.

Políticas sociales y género: una problematización del concepto de exclusión y participación social de las mujeres. In: TORQUINST, S. et al. Leituras de resistência: corpo, violência e poder. Florianópolis: Ed. Mulheres, 2009, p. 241-267.

LEON, M.; DEERE, C. La mujer y la política agraria en América Latina. Bogotá: Siglo XXI, 1986.

MEDEIROS, M.; COSTA, J. S. Is there a Feminization of Poverty in Latin America? World Development, v. 36, issue 1, p. 115-127, Jan. 2008.

MOGHADAM, V. The "Feminization of Poverty" and Women's Human Rights. SHS Papers in Women's studies/ Gender Research, n. 2, Jul. Paris: Unesco, 2005.

MOHANTY, C. Under Western Eyes. Feminist Scholarship and Colonial Discourses In: MOHANTY, C. et al. Third World Women and the Politics of Feminism. Indianapolis: Indiana University Press, 1987.
MOLYNEUX, M. Change and Continuity in Social Protection in Latin America: Mothers at the Service of the State? Unrisd Working Paper. Geneva: Unrisd, 2006.

MURGUIALDAY, C. Feminización de la pobreza. 2006. Disponible en: <http://www.dicc.hegoa.ehu.es/listar/

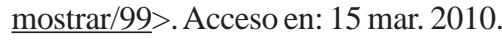

O’CONNOR, A. Poverty Knowledge. Social Science, Social Policy and the Poor in Twentieth-Century US History. New Jersey: Princeton University Press, 2001.

PEARCE, D. The Feminization of Poverty: Women, Work and Welfare. Urban and Social Change Review, Washington, v. 11, p. 28-36, 1978.

PNUD. Las dimensiones de la pobreza, 1997. Disponible en: <http://guiagenero.mzc.org.es/GuiaGeneroCache/ Pagina FeminiPobrez 000078.html>. Acceso en: 15 mar. 2010.

SCOTT, J. Gender: A Useful Category of Historical Analysis. American Historical Review, American Historical Association, v. 91, n. 5, p. 1053-1075, 1986.

\section{Notas}

1 Tesis de doctorado en curso "Una mirada genealogica de la 'domesticidad' a través de las políticas sociales" en Ciencias Sociales, Universidad de Buenos Aires, dirigida por la Dra. Estela Grassi y co dirigida por la Dra. Susana Murillo.

2 Se refiere sobre todo a la Aid to Families with Dependent Children (AFDC), vigente en los EEUU entre 1935 y 1996.

3 Especialmente el Banco Mundial y el PNUD. Para este último y sus contradicciones respecto de los diagnósticos de feminización, véase Damián(2003).

4 Aun tomando la perspectiva de la pobreza por ingresos, existen dificultades con la ausencia de datos y dificultades de desagregación. Este hecho se ha agravado en Argentina en los últimos años con la dificultad para acceder a estadísticas confiables, debido a la intervención y sucesivos cambios de metodología ocurridos en el Instituto Nacional de Estadística y Censos (Indec).

5 Cabe recordar que la advertencia de Scott (1986) acerca de la incorporación masiva de la categoría de género a los marcos institucionales, se relaciona con la "neutralidad" política de esta categoría en comparación con aquellas posiciones que se identifican más claramente como feministas.

6 Es interesante la observación de Pearce (1978) respecto de este carácter cultural de la jefatura del hogar y las consecuencias para la investigación. Plantea que mediante 
la identificación de la jefatura femenina se presupone, en algunos usos, una familia rota - fuera de la norma, y que la jefatura masculina presupone una familia completa con otro adulto a cargo. Ambas imágenes pueden ser inexactas.

\section{Paula Lucía Aguilar}

aguilarpl@gmail.com

Becaria doctoral del Consejo Nacional de Investigaciones Científicas y Técnicas de La República Argentina (Conicet) - Instituto de Investigaciones Gino Germani, Facultad de Ciencias Sociales (FSOC), Universidad de Buenos Aires (UBA)

Directora de Tesis: Dra. Estela Grassi

Co directora: Dra. Susana Murillo

\section{FSOC- UBA}

Instituto de Investigaciones Gino Germani

Facultad de Ciencias Sociales

Pte. J. E. Uriburu $9506^{\circ}$.

Buenos Aires - Argentina

Codigo Postal: 1114 